\title{
The asymptotic hazard rate of sums of discrete random variables
}

\author{
Joachim Arts ${ }^{\mathrm{a}}$, Geert-Jan van Houtum ${ }^{\mathrm{a}}$, Bert Zwart ${ }^{\mathrm{b}, *}$ \\ a Eindhoven University of Technology (TU/e), Department of Industrial Engineering, Netherlands \\ ${ }^{\mathrm{b}}$ CWI Amsterdam, Department of Mathematics at TU/e, Netherlands
}

\section{A R T I C L E I N F O}

\section{Article history:}

Received 4 February 2016

Received in revised form 30 January 2017

Accepted 3 February 2017

Available online 16 February 2017

\section{MSC:}

60E05

\section{Keywords:}

Hazard rates

Tail asymptotics

\section{A B S T R A C T}

We show that the asymptotic hazard rate of the sum of discrete random variables is dominated by the smallest asymptotic failure rate of the summands.

(c) 2017 Elsevier B.V. All rights reserved.

\section{Introduction and main result}

The purpose of this note is to determine the asymptotic hazard rate of the sum of independent non-negative discrete random variables. More precisely, we prove the following.

Proposition 1. Let $X, Y$ be independent non-negative integer-valued random variables such that $P(X=n) / P(X \geq n) \rightarrow r$ and $P(Y=n) / P(Y \geq n) \rightarrow s$ as $n \rightarrow \infty, r, s \in[0,1]$. As $n \rightarrow \infty$,

$$
P(X+Y=n) / P(X+Y \geq n) \rightarrow \min \{r, s\} .
$$

Proposition 1 is related to a classical convolution property of subexponential and related distributions; in particular Theorem 3 of Embrechts and Goldie (1980); more recent formulations can be found in Block et al. (2014, 2015). In this article, we focus on integer-valued distributions. Our motivation comes from an inventory model with lost sales in which ordered inventory arrives after a large lead time $n$ (Arts et al., in preparation). In that problem an approximation is developed that becomes accurate when $n$ is large, and the quality of the approximation turns out to depend on the result of the proposition presented here.

\section{Proof}

Without loss of generality, $r \leq s$. The proof will distinguish three cases: $r=1, r<s \leq 1$ and $r=s<1$.

\footnotetext{
* Corresponding author.

E-mail address: bertz@cwi.nl (B. Zwart).
} 
The case $r<s \leq 1$ : observe that the assumptions on $X$ and $Y$ are equivalent to

$$
\frac{P(X>n+m)}{P(X>n)} \rightarrow(1-r)^{m}, \quad \frac{P(Y>n+m)}{P(Y>n)} \rightarrow(1-s)^{m},
$$

as $n \rightarrow \infty$ along the integers, for every integer $m$. This should be compared with the class of distributions called $\mathscr{L}(\gamma)$, for $\gamma \geq 0$. A random variable $U$ is a member of $\mathcal{L}(\gamma)$ if, for all $y>0$

$$
\frac{P(U>x+y)}{P(U>x)} \rightarrow e^{-\gamma y},
$$

as $x \rightarrow \infty$ for all real $y$. Theorem 3 of Embrechts and Goldie (1980) essentially states, that if $U \in \mathscr{L}(\gamma)$ and $V \in \mathcal{L}(\delta)$ then $U+V \in \mathcal{L}(\min \{\gamma, \delta\})$.

Since our setting is restricted to integer-valued r.v.'s $X$ is not member of the class $\mathscr{L}(-\ln (1-r))$ a formal proof is needed. In addition, the inequality on the last line of page 253 of Embrechts and Goldie (1980) is not correct. In what follows we correct (and even simplify) that proof and have it apply to our discrete setting.

Set $F(n)=P(X \leq n), G(n)=P(Y \leq n), H(n)=P(X+Y \leq n), \bar{F}(n)=1-F(n) . \bar{G}$ and $\bar{H}$ are defined similarly. Observe now that, as in p. 252 of Embrechts and Goldie (1980):

$$
\begin{aligned}
0 & \leq \frac{P(X+Y>n ; Y>n-m)}{P(X+Y>n ; Y \leq n-m)} \leq \frac{P(Y>n-m)}{P(X>n ; Y \leq n-m)} \\
& =\frac{P(Y>n-m)}{P(X>n-m)} \frac{P(X>n-m)}{P(X>n)} \frac{P(X>n)}{P(X>n) P(Y \leq n-m)} \rightarrow 0 \times(1-r)^{-m} \times 1=0 .
\end{aligned}
$$

Let $f(n) \sim g(n)$ denote $f(n) / g(n) \rightarrow 1$. The above implies that

$$
P(X+Y>n) \sim \sum_{k=0}^{n-m} P(X>n-k) P(Y=k),
$$

so that also (replacing $m$ by $m-l$ and $n$ by $n-l$ )

$$
P(X+Y>n-l) \sim \sum_{k=0}^{n-m} \frac{P(X>n-k-l)}{P(X>n-k)} P(X>n-k) P(Y=k) .
$$

Set now $U_{X}(j, l)=\sup \{n: P(X>n-l) / P(X>n) ; n \geq j\}$ and $L_{X}(j, l)=\inf \{n: P(X>n-l) / P(X>n) ; n \geq j\}$. The r.h.s. of (3) is between

$$
L_{X}(m, l) \sum_{k=0}^{n-m} P(X>n-k) P(Y=k) \quad \text { and } \quad U_{X}(m, l) \sum_{k=0}^{n-m} P(X>n-k) P(Y=k) .
$$

Since $\lim _{m \rightarrow \infty} L_{X}(m, l)=\lim _{m \rightarrow \infty} U_{X}(m, l)=(1-r)^{-l}$, we obtain from (3),

$$
P(X+Y>n-l) \sim(1-r)^{-l} \sum_{k=0}^{n-m} P(X>n-k) P(Y=k) \sim(1-r)^{-l} P(X+Y>n) .
$$

The last equivalence follows from (2).

The case $r=s<1$ : define $L_{Y}$ and $U_{Y}$ similarly as $L_{X}$ and $U_{X}$. As (2.12) of Embrechts and Goldie (1980) is also valid for integer-valued r.v. we simply state its conclusion. For any $m>0$ :

$$
P(X+Y>n-l) \leq \max \left\{U_{X}(m, l), U_{Y}(n-m+l, l)\right\} P(X+Y>n) .
$$

Thus, we see that

$$
\limsup _{n \rightarrow \infty} \frac{P(X+Y>n-l)}{P(X+Y>n)} \leq \max \left\{U_{X}(m, l),(1-r)^{-l}\right\},
$$

for every $m>0$. Now let $m \rightarrow \infty$ to conclude that

$$
\limsup _{n \rightarrow \infty} \frac{P(X+Y>n-l)}{P(X+Y>n)} \leq(1-r)^{-l} .
$$

The proof of the lower bound is similar.

The case $r=1$ : let $\epsilon \in(0,1)$ and $K$ be such that $P(X=i) \geq(1-\epsilon) P(X \geq i)$ for $i \geq K$. Write for $n>2 K$ :

$$
\begin{aligned}
P(X+Y=n) & \geq \sum_{i=K}^{n} P(X=i) P(Y=n-i) \geq(1-\epsilon) \sum_{i=K}^{n} P(X \geq i) P(Y=n-i) \\
& \geq(1-\epsilon) P(X+Y \geq n ; Y \leq n-K)=(1-\epsilon)[P(X+Y \geq n)-P(X+Y \geq n ; Y>n-K)] \\
& \geq(1-\epsilon)[P(X+Y \geq n)-P(Y>n-K)] .
\end{aligned}
$$


Thus,

$$
1 \geq \frac{P(X+Y=n)}{P(X+Y \geq n)} \geq(1-\epsilon)\left[1-\frac{P(Y>n-K)}{P(X+Y \geq n)}\right] .
$$

Since

$$
\frac{P(Y>n-K)}{P(X+Y \geq n)} \leq \frac{P(Y>n-K)}{P(Y>n-K-1) P(X<K+1)} \rightarrow 0
$$

given the assumption on $Y$, we conclude that

$$
\liminf _{n \rightarrow \infty} \frac{P(X+Y=n)}{P(X+Y \geq n)} \geq 1-\epsilon .
$$

The proof is now complete by letting $\epsilon \downarrow 0$.

\section{Acknowledgements}

The authors are grateful to a referee for a careful reading of the manuscript. The research of the last author is supported by VICI grant 639.033 .413 of NWO.

\section{References}

Arts, J., Houtum, G.J., Levi, R., 2017. Base-stock policies for lost sales models: aggregation and asymptotics (in preparation).

Block, H.W., Langberg, N.A., Savits, T.H., 2014. The limiting failure rate for a convolution of gamma distributions. Statist. Probab. Lett. 94 , 176-180.

Block, H.W., Langberg, N.A., Savits, T.H., 2015. The limiting failure rate for a convolution of life distributions. J. Appl. Probab. 52, 894-898.

Embrechts, P., Goldie, C., 1980. On closure and factorization properties of subexponential and related distributions. J. Aust. Math. Soc. A 29, 243-256. 